\title{
A Synthesis of Novel Pyrazolylthienopyrimidine Derivatives as IL-6/STAT3 Inhibitors
}

\author{
Sung Min Kim, Seung Woong Lee, and Yang-Heon Song* \\ Department of Chemistry, Mokwon University, Doanbuk-ro 88, Daejeon 302-729, Korea. *E-mail: yhsong@mokwon.ac.kr \\ Received April 22, 2014, Accepted May 26, 2014
}

Key Words : Thienopyrimidine, Pyrazole, Interleukin-6, STAT-3

The interleukin-6 (IL-6) as a pro-inflammatory cytokine with TNF- $\alpha$ and IL-8 plays a central role in the induction of inflammation as well as in the continuation of a chronic inflammatory immune response. ${ }^{1}$ And, an over-production of IL-6 have been found in inflammatory tissues of several diseases, such as rheumatoid arthritis, psoriasis, inflammatory bowel disease, osteoarthritis, multiple myeloma, and also in human atherosclerotic plaque. ${ }^{2}$ IL- 6 binds to its receptor (IL-6R, a ligand-binding $80 \mathrm{kDa}$ glycoprotein chain) and induces the homodimerization of a signal transducing glycoprotein 130 (gp130), leading to the activation of the Janus kinase (Jak)/signal transducer and signal activator of transcription-3 (STAT3). ${ }^{3}$ STAT3 is also frequently overexpressed or persistently activated in most tumors and cancer, and activated STAT3 was found to suppress tumorimmune surveillance. ${ }^{4}$ Therefore, the blockade of STAT3 activation pathway stimulated by IL- 6 could be an attractive therapeutic target for discovery of new drugs and is currently under intense investigation. ${ }^{5}$

In the other hand, thienopyrimidine and their derivatives are an important class of the most biologically active compounds, having diverse activities such as antibacterial, analgesic, antitumor, antioxidant, P2Y12 platelet aggregation inhibitor, and Aurora kinase inhibitor. ${ }^{6}$ We have synthesized over the years thienopyrimidine derivatives of promising biological activity. ${ }^{7}$ As part of a programme to discover novel inhibitors using thienopyrimidines, some of these compounds were found to possess potent IL-6/STAT3 inhibition. ${ }^{8}$ This result encouraged us to prepare new thienopyrimidines having pyrazole moiety in attempt to improve the IL-6/STAT3 inhibitory activity.

Pyrazoles have been also reported to have a wide range of biological properties. ${ }^{9}$ Moreover, incorporation of pyrazole moiety into other heterocycles gave various pharmacological activities. ${ }^{10}$ Although a lot of methodologies have been developed over the years, the regiocontrolled and practical synthesis of 1,5-pyrazoles ${ }^{11}$ over 1,3-pyrazole is still less literature precedent and remains a noteworthy challenge. To the best of our knowledge no report have so far made in the synthesis of regioselective pyrazolylthienopyrimidine by the one pot reaction of $(E)$-3-(dimethylamino)-1-phenylprop-2en-1-one 2 with 4-hydrazinothienopyrimidine 1 . Herein we report the efficient synthesis and biological activity of novel pyrazolylthienopyrimidine derivatives, $\mathbf{3 A}$ and $\mathbf{3 B}$, as IL-6/ STAT3 inhibitor.

The required reactant 2 can be easily prepared by the
Table 1. Optimization for the reaction of $\mathbf{1 A}$ with $\mathbf{2 a}$

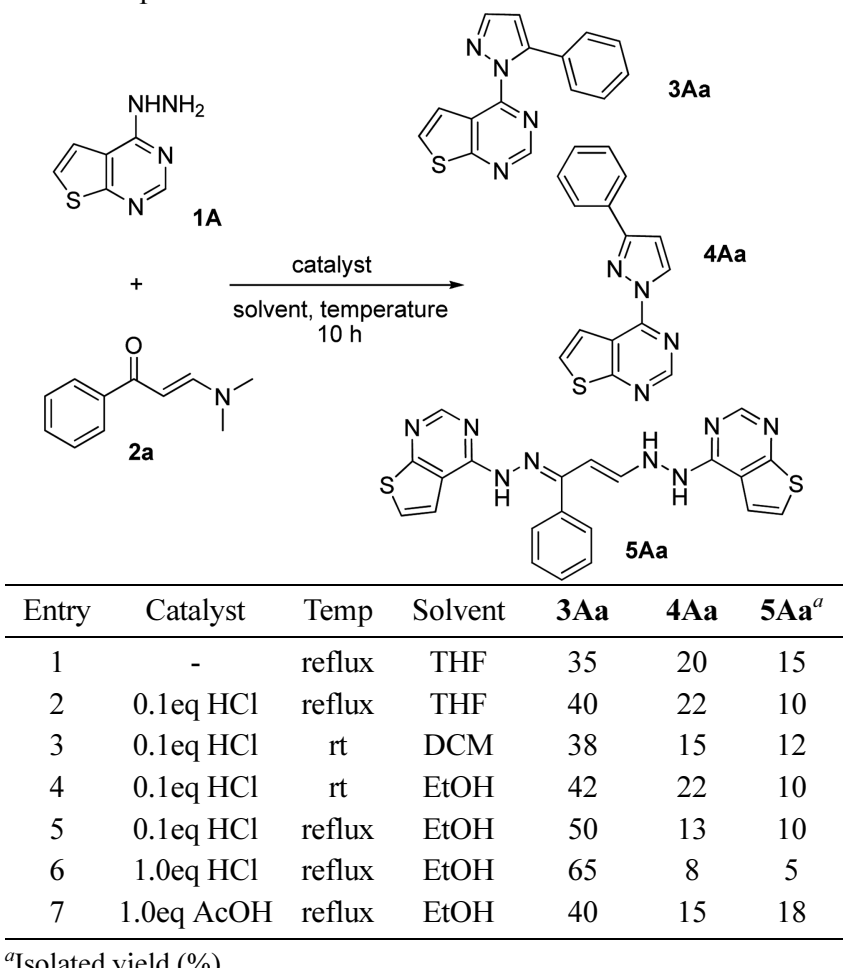

reaction of acetophenone with $N, N$-dimethylformamide dimethyl acetal (DMFDMA) in refluxing xylene. The reactants, $\mathbf{1 A}$ and 1B, were also obtained according to the procedure we have previously reported..$^{7 f}$ In our initial studies toward synthesis of substituted pyrazolylthienopyrimidine, (E)-3-(dimethylamino)-1-phenylprop-2-en-1-one (2a) was reacted with 4-hydrazinothieno[2,3- $d]$ pyrimidine (1A) in $\mathrm{THF}$ at reflux for $10 \mathrm{~h}$. It was found that three products from the reaction mixture were formed, respectively, 4-(5-phenyl1H-pyrazol-1-yl)thieno[2,3-d]pyrimidine (3Aa, 1,5-isomer, 35\%), 4-(3-phenyl-1H-pyrazol-1-yl)thieno[2,3- $d]$ pyrimidine (4Aa, 1,3-isomer, 20\%) and 1,2-adduct (5Aa, 15\%), as shown Table 1. Accordingly, reaction optimization for the regioselective formation of 1,5-isomer was investigated at the various conditions (catalyst, reaction temperature and solvent). When the same reaction was carried out in refluxing ethanol in the presence of 1 equiv $\mathrm{HCl}$ as a catalyst, 3Aa was obtained as a major product in optimum yield $(65 \%$, Table 1 , entry 6) within $6 \mathrm{~h}$.

Next, as depicted in Table 2, the reaction of $\mathbf{1 A}$ with $\mathbf{2} \mathbf{b}-\mathbf{h}$ 
Table 2. Synthesis of phenylpyrazolylthienopyrimidine $\mathbf{3 A}$ and $\mathbf{3 B}$

\begin{tabular}{cccccc} 
& & & & \\
\hline
\end{tabular}

${ }^{a}$ Isolated yield (\%).

was further evaluated under optimized reaction condition. According to the LC-MS analysis of the reaction mixture, major products $\mathbf{3} \mathbf{A b}-\mathbf{h}$ were obtained in good yields with small amount (4-10\%) of $\mathbf{4}$ and $\mathbf{5}$, and no effort was performed to isolate the side products (Table 2). The results also indicated that $\mathbf{2 b - d}$ having electron-donating group on phenyl ring gave products in slightly better yields (Table 2, entries 2-4) as compared with 2e-h having electron-withdrawing group (entries 5-8). The reaction of $\mathbf{1 B}$ and $\mathbf{2 a - h}$ was also successfully applied to the synthesis of $\mathbf{3 B a}-\mathbf{h}$ as a major product. Notably, the reaction of $\mathbf{2 e - h}$, having electron-withdrawing groups, with $\mathbf{1 B}$ afforded $\mathbf{3 B e}-\mathbf{h}$ in good yields along with slightly increased 1,2-adduct, 5Be-h (data was not shown). To provide an evidence for the assigned structure $\mathbf{3 A}$ and $\mathbf{4 A}$, an authentic sample of $4 \mathbf{A a}$ was prepared by an alternative synthesis ${ }^{12}$ as shown Scheme 1 . This was identical in all respects ( $\mathrm{mp}, \mathrm{IR},{ }^{1} \mathrm{H} \mathrm{NMR}$, and MS spectra) with 4Aa that is isolated from the reaction of $\mathbf{1 A}$ with 2a. It is noteworthy that the $\mathrm{H}-4$ of pyrazole ring in ${ }^{1} \mathrm{H}$ NMR spectrum of $\mathbf{4 A a}$ appeared as a doublet at $\delta 7.10$ with $J_{4,5}=2.8 \mathrm{~Hz}$ whereas a doublet for the $\mathrm{H}-4$ of pyrazole ring in $3 \mathrm{Aa}$ was found at $\delta 6.90$ with $J_{3,4}=1.6 \mathrm{~Hz}^{11}$

The newly synthesized phenylpyrazolylthienopyrimidine derivatives 3Aa-h and 3Ba-h were evaluated for their inhibitory activities against STAT3 activation by IL-6, according to the reported method. ${ }^{13,14}$ Table 3 lists the biological

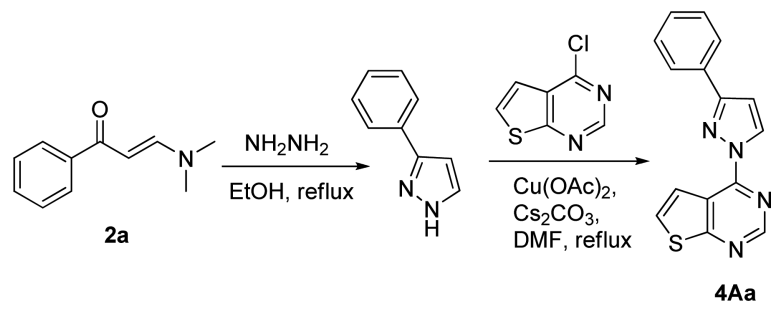

Scheme 1. Alternative synthesis of 4Aa.

Table 3. Inhibitory effects of compounds $\mathbf{3 A}$ and $\mathbf{3 B}$ on IL-6induced Stat3 activation ${ }^{a}$

\begin{tabular}{cccc}
\hline Compounds & $\mathrm{IC}_{50}(\mu \mathrm{M})^{b}$ & Compounds & $\mathrm{IC}_{50}(\mu \mathrm{M})^{b}$ \\
\hline 3Aa & 3.6 & $\mathbf{3 B a}$ & $>100$ \\
3Ab & 1.7 & $\mathbf{3 B b}$ & 30.3 \\
3Ac & 3.2 & $\mathbf{3 B c}$ & 43.9 \\
$\mathbf{3 A d}$ & 1.5 & $\mathbf{3 B d}$ & 37.2 \\
3Ae & 20.6 & $\mathbf{3 B e}$ & 25.5 \\
$\mathbf{3 A f}$ & 12.6 & $\mathbf{3 B f}$ & 15.1 \\
$\mathbf{3 A g}$ & 9.2 & $\mathbf{3 B g}$ & 3.4 \\
$\mathbf{3 A h}$ & 15.8 & $\mathbf{3 B h}$ & 14.9 \\
Genistein $^{c}$ & 15 & &
\end{tabular}

${ }^{a}$ Data ate mean \pm standard error values of three replications. ${ }^{b} \mathrm{IC}_{50}$ : mean $(50 \%)$ value of inhibition concentration. ${ }^{c}$ Genistein was used as a positive control.

data for compounds 3Aa-h and 3Ba-h. Compound 3Aa identified from the initial screening exhibited strong inhibitory activity with an $\mathrm{IC}_{50}$ value of $3.6 \mu \mathrm{M}$ on IL-6-induced STAT3 activation. Other compounds $\mathbf{3 A b}-\mathbf{h}$ showed moderate to strong inhibition in the range of $1.5-20.6 \mu \mathrm{M}$. It is noteworthy that the most potent inhibitors, $\mathbf{3 A b}$ and $\mathbf{3 A d}\left(\mathrm{IC}_{50}\right.$ : 1.5 and $1.7 \mu \mathrm{M}$ ), have alkyl group at para position of benzene moiety. Minor compounds $\mathbf{4}$ showed less inhibitory activity than compounds $\mathbf{3}$. None of the compounds tested had any cytotoxicity in Hep3B cells with MTT assay at these concentrations (data not shown). Further studies on the biological activities of these compounds are under way.

In summary, a new class of phenylpyrazolylthienopyrimidine derivatives were prepared as a main product by the reaction of $(E)$-3-(dimethylamino)-1-phenylprop-2-en-1-one and 4-hydrazinothienopyrimidine in good yields. Among them, two compounds, $\mathbf{3 A b}$ and $\mathbf{3 A d}$, showed the most potent inhibitory activity on the Stat3-dependent luciferase assay compared with genistein as a positive control.

\section{Experimental}

Chemistry. Melting points were determined in capillary tubes on Büchi apparatus and are uncorrected. Each compound of the reactions was checked on thin-layer chromatography of Merck Kieselgel $60 \mathrm{~F}_{254}$ and purified by column chromatography Merck silica gel (70-230 mesh). The ${ }^{1} \mathrm{H}$ NMR spectra were recorded on Unity Inova 400NB FT NMR spectrometer $\left(400 \mathrm{MHz}\right.$ ) with $\mathrm{Me}_{4} \mathrm{Si}$ as internal standard and chemical shifts are given in ppm $(\delta)$. Mass spectra were recorded on a HP 59580 B spectrometer. Elemental analyses 
were performed on a Carlo Erba 1106 elemental analyzer.

General Procedure for the Preparation of $3 A$ and $3 B$. 4-Hydrazinothienopyrimidine $\mathbf{1 A}$ or $\mathbf{1 B}(10 \mathrm{mmol})$ and the appropriate $(E)-3$-(dimethylamino)-1-phenylprop-2-en-1one $2(10 \mathrm{mmol})$ were mixed in ethanol $(50 \mathrm{~mL})$ containing $\mathrm{HCl}$ (1 eq), and the mixture was heated to $70{ }^{\circ} \mathrm{C}$ for $6 \mathrm{~h}$. After evaporation of the solvent, the residue was purified by column chromatography using as eluent $\mathrm{CH}_{2} \mathrm{Cl}_{2}$-EtOAc (8:2) to give $\mathbf{3 A}$ and $\mathbf{3 B}$, respectively.

4-(5-Phenyl-1 $H$-pyrazol-1-yl)thieno[2,3- $d]$ pyrimidine (3Aa). Yield 65\%; mp 54-55 ${ }^{\circ} \mathrm{C} ;{ }^{1} \mathrm{H}$ NMR (DMSO- $d_{6}$ ) $\delta$ 8.89 (s, $1 \mathrm{H}$, pyrimidine), $8.62(\mathrm{~d}, 1 \mathrm{H}, J=5.9 \mathrm{~Hz}$, thiophene), 8.36 (d, $1 \mathrm{H}, J=1.6 \mathrm{~Hz}, \mathrm{H}-3$, pyrazole), 7.97 (d, 2H, phenyl), 7.60 (d, 1H, $J=5.9 \mathrm{~Hz}$, thiophene), 7.48 (m, 3H, phenyl), 6.90 (d, 1H, $J=1.6 \mathrm{~Hz}, \mathrm{H}-4$, pyrazole); MS (ESI): $(\mathrm{m} / \mathrm{z})$ $278.93\left(\mathrm{M}^{+}\right)$. Anal. Calcd. For $\mathrm{C}_{15} \mathrm{H}_{10} \mathrm{~N}_{4} \mathrm{~S}$ : C, 64.73; H, 3.62; N, 20.13. Found: C, 64.54; H, 3.50; N, 20.27.

4-(5-(4-Ethylphenyl-1H-pyrazol-1-yl)thieno[2,3-d]pyrimidine (3Ab). Yield 76\%; mp 135-136 ${ }^{\circ} \mathrm{C}$; ${ }^{1} \mathrm{H}$ NMR (DMSO$\left.d_{6}\right) \delta 8.45$ (s, 1H, pyrimidine), $7.92(\mathrm{~d}, 1 \mathrm{H}, J=1.6 \mathrm{~Hz}, \mathrm{H}-3$, pyrazole), 7.70 (d, 2H, phenyl), $7.62(\mathrm{~d}, 1 \mathrm{H}, J=5.9 \mathrm{~Hz}$, thiophene), 7.47 (d, $1 \mathrm{H}, J=5.9 \mathrm{~Hz}$, thiophene), 7.30 (d, 2H, phenyl), 6.79 (d, 1H, J=1.6 Hz, H-4, pyrazole), 2.63 (q, 2H, $\left.\mathrm{CH}_{2}\right), 1.17$ (t, 3H, Me); MS (ESI): (m/z) $306.95\left(\mathrm{M}^{+}\right)$. Anal. Calcd. For $\mathrm{C}_{17} \mathrm{H}_{14} \mathrm{~N}_{4} \mathrm{~S}$ : C, 66.64; H, 4.61; N, 18.29. Found: C, 66.48; H, 4.50; N, 18.44 .

4-(5-m-Tolyl-1 $H$-pyrazol-1-yl)thieno[2,3-d]pyrimidine (3Ac). Yield 70\%; mp 162-163 ${ }^{\circ} \mathrm{C} ;{ }^{1} \mathrm{H}$ NMR (DMSO- $\left.d_{6}\right) \delta$ 8.50 (s, $1 \mathrm{H}$, pyrimidine), 8.05 (d, $1 \mathrm{H}, J=6.0 \mathrm{~Hz}$, thiophene), 7.96 (d, 1H, $J=1.6 \mathrm{~Hz}, \mathrm{H}-3$, pyrazole), 7.72 (s, 1H, phenyl, $\left.\mathrm{H}_{2}{ }^{\prime}\right), 7.67\left(\mathrm{~d}, 1 \mathrm{H}\right.$, phenyl, $\left.\mathrm{H}_{6}{ }^{\prime}\right), 7.54(\mathrm{~d}, 1 \mathrm{H}, J=5.9 \mathrm{~Hz}$, thiophene), $7.32\left(\mathrm{t}, 1 \mathrm{H}\right.$, phenyl, $\left.\mathrm{H}_{5}^{\prime}\right), 7.10\left(\mathrm{~d}, 1 \mathrm{H}\right.$, phenyl, $\left.\mathrm{H}_{4}^{\prime}\right)$, 6.89 (d, 1H, $J=1.6 \mathrm{~Hz}, \mathrm{H}-4$, pyrazole), 2.26 (s, 3H, Me); MS (ESI): $(m / z)$ 292.79. $\left(\mathrm{M}^{+}\right)$. Anal. Calcd. For $\mathrm{C}_{16} \mathrm{H}_{12} \mathrm{~N}_{4} \mathrm{~S}$ : C, 65.73; H, 4.14; N, 19.16. Found: C, 65.70; H, 4.05; N, 19.02 .

4-(5-p-Tolyl-1 $H$-pyrazol-1-yl)thieno[2,3-d]pyrimidine (3Ad). Yield 78\%; mp 164-165 ${ }^{\circ} \mathrm{C} ;{ }^{1} \mathrm{H}$ NMR (DMSO- $\left.d_{6}\right) \delta$ 8.47 (s, 1H, pyrimidine), 8.17 (d, $1 \mathrm{H}, J=6.0 \mathrm{~Hz}$, thiophene), 7.88 (d, 1H, $J=1.6 \mathrm{~Hz}, \mathrm{H}-3$, pyrazole), 7.82 (d, 1H, $J=5.9$ $\mathrm{Hz}$, thiophene), 7.74 (d, 2H, phenyl), 7.24 (d, 2H), 6.77 (d, $1 \mathrm{H}, J=1.6 \mathrm{~Hz}, \mathrm{H}-4$, pyrazole), 2.20 (s, 3H, Me); MS (ESI): $(m / z)$ 292.66. $\left(\mathrm{M}^{+}\right)$. Anal. Calcd. For $\mathrm{C}_{16} \mathrm{H}_{12} \mathrm{~N}_{4} \mathrm{~S}$ : C, 65.73; H, 4.14; N, 19.16. Found: C, 65.57; H, 4.11; N, 19.27.

4-(5-3-Bromophenyl-1H-pyrazol-1-yl)thieno[2,3-d]pyrimidine (3Ae). Yield 60\%; mp 142-143 ${ }^{\circ} \mathrm{C}$; ${ }^{1} \mathrm{H}$ NMR (DMSOd6) $\delta 8.48$ (s, 1H, pyrimidine), 7.98 (d, 1H, J=1.6 Hz, H-3, pyrazole), 7.90 (d, 1H, $J=5.9 \mathrm{~Hz}$, thiophene), 7.74 (d, 1H, $J$ $=5.9 \mathrm{~Hz}$, thiophene), $7.47\left(\mathrm{~s}, 1 \mathrm{H}\right.$, phenyl, $\left.\mathrm{H}_{2}{ }^{\prime}\right), 7.42-7.35(\mathrm{~m}$, $3 \mathrm{H}), 6.78$ (d, 1H, $J=1.6 \mathrm{~Hz}, \mathrm{H}-4$, pyrazole); MS (ESI): $(\mathrm{m} / \mathrm{z})$ 358.97. $\left(\mathrm{M}^{+}\right)$. Anal. Calcd. For $\mathrm{C}_{15} \mathrm{H}_{9} \mathrm{BrN}_{4} \mathrm{~S}$ : C, 50.43; H, 2.54 ; N, 15.68. Found: C, 50.20; H, 2.42; N, 15.88 .

4-(5-4-Bromophenyl-1H-pyrazol-1-yl)thieno[2,3-d]pyrimidine (3Af). Yield 66\%; mp 85-86 ${ }^{\circ} \mathrm{C}$; ${ }^{1} \mathrm{H}$ NMR (DMSO$\left.d_{6}\right) \delta 8.76(\mathrm{~s}, 1 \mathrm{H}$, pyrimidine $), 8.10(\mathrm{~d}, 1 \mathrm{H}, J=5.9 \mathrm{~Hz}$, thiophene), 7.95 (d, 1H, $J=1.6 \mathrm{~Hz}, \mathrm{H}-3$, pyrazole), 7.82 (d, $1 \mathrm{H}, J=5.9 \mathrm{~Hz}$, thiophene), $7.54(\mathrm{~d}, 2 \mathrm{H}$, phenyl), $7.28(\mathrm{~d}, 2 \mathrm{H}$, phenyl), 6.78 (d, 1H, $J=1.6 \mathrm{~Hz}, \mathrm{H}-4$, pyrazole); MS (ESI): $(\mathrm{m} / z)$ 358.81. $\left(\mathrm{M}^{+}\right)$. Anal. Calcd. For $\mathrm{C}_{15} \mathrm{H}_{9} \mathrm{BrN}_{4} \mathrm{~S}$ : C, 50.43; H, 2.54; N, 15.68. Found: C, 50.31; H, 2.42; N, 15.61.

4-(5-3-Chlorophenyl-1H-pyrazol-1-yl)thieno[2,3-d]pyrimidine (3Ag). Yield 63\%; mp 168-169 ${ }^{\circ} \mathrm{C}$; ${ }^{1} \mathrm{H}$ NMR (DMSO$\left.d_{6}\right) \delta 8.48(\mathrm{~s}, 1 \mathrm{H}$, pyrimidine), $7.98(\mathrm{~d}, 1 \mathrm{H}, J=1.6 \mathrm{~Hz}, \mathrm{H}-3$, pyrazole), 7.74 (d, $1 \mathrm{H}, J=5.9 \mathrm{~Hz}$, thiophene), 7.57 (d, 1H, $J$ $=5.9 \mathrm{~Hz}$, thiophene), $7.47\left(\mathrm{~s}, 1 \mathrm{H}\right.$, phenyl, $\left.\mathrm{H}_{2}{ }^{\prime}\right), 7.45-7.39(\mathrm{~m}$, $3 \mathrm{H}), 6.76$ (d, 1H, $J=1.6 \mathrm{~Hz}, \mathrm{H}-4$, pyrazole); MS (ESI): $(\mathrm{m} / \mathrm{z})$ $312.95\left(\mathrm{M}^{+}\right)$. Anal. Calcd. For $\mathrm{C}_{15} \mathrm{H}_{9} \mathrm{ClN}_{4} \mathrm{~S}$ : C, 57.60; H, 2.90; N, 17.91. Found: C, 57.69; H, 2.79; N, 17.77.

4-(5-4-Chlorophenyl-1 H-pyrazol-1-yl)thieno[2,3-d]pyrimidine (3Ah). Yield 65\%; mp 172-173 ${ }^{\circ} \mathrm{C}$; ${ }^{1} \mathrm{H}$ NMR (DMSO$\left.d_{6}\right) \delta 8.76(\mathrm{~s}, 1 \mathrm{H}$, pyrimidine), $8.09(\mathrm{~d}, 1 \mathrm{H}, J=5.9 \mathrm{~Hz}$, thiophene), 8.02 (d, 1H, J=1.6 Hz, H-3, pyrazole), 7.89 (d, $1 \mathrm{H}, J=5.9 \mathrm{~Hz}$, thiophene), 7.49 (d, 2H, phenyl), $7.35(\mathrm{~d}, 2 \mathrm{H}$, phenyl), 6.87 (d, 1H, $J=1.6 \mathrm{~Hz}, \mathrm{H}-4$, pyrazole); MS (ESI): $(\mathrm{m} / z) 312.87\left(\mathrm{M}^{+}\right)$. Anal. Calcd. For $\mathrm{C}_{15} \mathrm{H}_{9} \mathrm{ClN}_{4} \mathrm{~S}$ : C, 57.60; H, 2.90; N, 17.91. Found: C, 57.44; H, 2.82; N, 18.14.

4-(5-Phenyl-1 $H$-pyrazol-1-yl)thieno[3,2-d]pyrimidine (3Ba). Yield 62\%; mp 91-93 ${ }^{\circ} \mathrm{C} ;{ }^{1} \mathrm{H}$ NMR (DMSO- $\left.d_{6}\right) \delta$ 8.56 (s, 1H, pyrimidine), 8.33 (d, $1 \mathrm{H}, J=1.6 \mathrm{~Hz}, \mathrm{H}-3$, pyrazole), 8.31 (d, $1 \mathrm{H}, J=5.9 \mathrm{~Hz}$, thiophene), 7.80 (d, 2H, phenyl), $7.46(\mathrm{~m}, 3 \mathrm{H}$, phenyl), 7.38 (d, 1H, $J=5.9 \mathrm{~Hz}$, thiophene), 6.84 (d, 1H, $J=1.6 \mathrm{~Hz}, \mathrm{H}-4$, pyrazole); MS (ESI): $(\mathrm{m} / \mathrm{z})$ $278.95\left(\mathrm{M}^{+}\right)$. Anal. Calcd. For $\mathrm{C}_{15} \mathrm{H}_{10} \mathrm{~N}_{4} \mathrm{~S}$ : C, 64.73; H, 3.62; N, 20.13. Found: C, 64.81; H, 3.52; N, 20.02.

4-(5-(4-Ethylphenyl-1H-pyrazol-1-yl)thieno[3,2-d]pyrimidine (3Bb). Yield 72\%; mp 177-178 ${ }^{\circ} \mathrm{C}$; ${ }^{1} \mathrm{H}$ NMR (DMSO$\left.d_{6}\right) \delta 8.69(\mathrm{~s}, 1 \mathrm{H}$, pyrimidine), $8.06(\mathrm{~d}, 1 \mathrm{H}, J=5.9 \mathrm{~Hz}$, thiophene), 7.85 (d, $1 \mathrm{H}, J=1.6 \mathrm{~Hz}, \mathrm{H}-3$, pyrazole), 7.63 (d, $2 \mathrm{H}$, phenyl), $7.61(\mathrm{~d}, 1 \mathrm{H}, J=5.9 \mathrm{~Hz}$, thiophene), $7.31(\mathrm{~d}, 2 \mathrm{H}$, phenyl), 6.50 (d, 1H, $J=1.6 \mathrm{~Hz}, \mathrm{H}-4$, pyrazole), 2.70 (q, 2H, $\left.\mathrm{CH}_{2}\right), 1.26$ (t, 3H, Me); MS (ESI): $(\mathrm{m} / \mathrm{z}) 306.90\left(\mathrm{M}^{+}\right)$. Anal. Calcd. For $\mathrm{C}_{17} \mathrm{H}_{14} \mathrm{~N}_{4} \mathrm{~S}$ : C, 66.64; H, 4.61; N, 18.29. Found: C, 66.52; H, 4.68; N, 18.18 .

4-(5-m-Tolyl-1H-pyrazol-1-yl)thieno[3,2-d]pyrimidine (3Bc). Yield 76\%; mp 111-112 ${ }^{\circ} \mathrm{C} ;{ }^{1} \mathrm{H}$ NMR (DMSO- $d_{6}$ ) $\delta$ 8.67 (s, $1 \mathrm{H}$, pyrimidine), $8.01(\mathrm{~d}, 1 \mathrm{H}, J=6.0 \mathrm{~Hz}$, thiophene), 7.95 (d, 1H, J=1.6 Hz, H-3, pyrazole), 7.73 (s, 1H, phenyl, $\left.\mathrm{H}_{2}{ }^{\prime}\right), 7.60\left(\mathrm{~d}, 1 \mathrm{H}\right.$, phenyl, $\left.\mathrm{H}_{6}{ }^{\prime}\right), 7.50(\mathrm{~d}, 1 \mathrm{H}, J=5.9 \mathrm{~Hz}$, thiophene), 7.28 (t, 1H, phenyl, $\left.\mathrm{H}_{5}^{\prime}\right), 7.18$ (d, 1H, phenyl, $\left.\mathrm{H}_{4}{ }^{\prime}\right), 6.85$ (d, 1H, $J=1.6 \mathrm{~Hz}, \mathrm{H}-4$, pyrazole), 2.32 (s, 3H, Me); MS (ESI): $(m / z)$ 292.92. $\left(\mathrm{M}^{+}\right)$. Anal. Calcd. For $\mathrm{C}_{16} \mathrm{H}_{12} \mathrm{~N}_{4} \mathrm{~S}$ : C, 65.73; H, 4.14; N, 19.16. Found: C, 65.61; H, 4.00; N, 19.11.

4-(5-p-Tolyl-1 $H$-pyrazol-1-yl)thieno[3,2-d]pyrimidine (3Bd). Yield 70\%; mp 131-132 ${ }^{\circ} \mathrm{C} ;{ }^{1} \mathrm{H}$ NMR (DMSO- $d_{6}$ ) $\delta$ 8.47 (s, 1H, pyrimidine), 7.83 (d, $1 \mathrm{H}, J=1.6 \mathrm{~Hz}, \mathrm{H}-3$, pyrazole), 7.74 (d, 2H, phenyl), $7.70(\mathrm{~d}, 1 \mathrm{H}, J=6.0 \mathrm{~Hz}$, thiophene), $7.40(\mathrm{~d}, 1 \mathrm{H}, J=5.9 \mathrm{~Hz}$, thiophene), $7.28(\mathrm{~d}, 2 \mathrm{H}), 6.75$ (d, $1 \mathrm{H}, J=1.6 \mathrm{~Hz}, \mathrm{H}-4$, pyrazole), 2.20 (s, 3H, Me); MS (ESI): $(m / z)$ 292.67. $\left(\mathrm{M}^{+}\right)$. Anal. Calcd. For $\mathrm{C}_{16} \mathrm{H}_{12} \mathrm{~N}_{4} \mathrm{~S}: \mathrm{C}$, 65.73; H, 4.14; N, 19.16. Found: C, 65.68; H, 4.22; N, 19.02 .

4-(5-3-Bromophenyl-1H-pyrazol-1-yl)thieno[3,2-d]pyrimidine (3Be). Yield 66\%; mp 111-113 ${ }^{\circ} \mathrm{C} ;{ }^{1} \mathrm{H}$ NMR (DMSO$\left.d_{6}\right) \delta 8.55$ (s, $1 \mathrm{H}$, pyrimidine), $8.02(\mathrm{~d}, 1 \mathrm{H}, J=1.6 \mathrm{~Hz}, \mathrm{H}-3$, 
pyrazole), $7.84(\mathrm{~d}, 1 \mathrm{H}, J=5.9 \mathrm{~Hz}$, thiophene), $7.78(\mathrm{~d}, 1 \mathrm{H}, J$ $=5.9 \mathrm{~Hz}$, thiophene), $7.49\left(\mathrm{~s}, 1 \mathrm{H}\right.$, phenyl, $\left.\mathrm{H}_{2}{ }^{\prime}\right), 7.40-7.35(\mathrm{~m}$, $3 \mathrm{H}), 6.76$ (d, 1H, $J=1.6 \mathrm{~Hz}, \mathrm{H}-4$, pyrazole); MS (ESI): $(\mathrm{m} / \mathrm{z})$ 358.86. $\left(\mathrm{M}^{+}\right)$. Anal. Calcd. For $\mathrm{C}_{15} \mathrm{H}_{9} \mathrm{BrN}_{4} \mathrm{~S}$ : C, 50.43; $\mathrm{H}$, $2.54 ;$ N, 15.68. Found: C, 50.57; H, 2.40; N, 15.49 .

4-(5-4-Bromophenyl-1 H-pyrazol-1-yl)thieno[3,2-d]pyrimidine (3Bf). Yield 65\%; mp 92-95 ${ }^{\circ} \mathrm{C}$; ${ }^{1} \mathrm{H}$ NMR (DMSO$\left.d_{6}\right) \delta 8.64(\mathrm{~s}, 1 \mathrm{H}$, pyrimidine $), 8.48(\mathrm{~d}, 1 \mathrm{H}, J=5.9 \mathrm{~Hz}$, thiophene), 8.02 (d, 1H, $J=1.6 \mathrm{~Hz}, \mathrm{H}-3$, pyrazole), 7.57 (d, $1 \mathrm{H}, J=5.9 \mathrm{~Hz}$, thiophene), 7.51 (d, 2H, phenyl), $7.33(\mathrm{~d}, 2 \mathrm{H}$, phenyl), 6.74 (d, 1H, $J=1.6 \mathrm{~Hz}, \mathrm{H}-4$, pyrazole); MS (ESI): $(\mathrm{m} / \mathrm{z})$ 358.48. $\left(\mathrm{M}^{+}\right)$. Anal. Calcd. For $\mathrm{C}_{15} \mathrm{H}_{9} \mathrm{BrN}_{4} \mathrm{~S}: \mathrm{C}, 50.43$; H, 2.54; N, 15.68. Found: C, 50.30; H, 2.48; N, 15.54 .

4-(5-3-Chlorophenyl-1 H-pyrazol-1-yl)thieno[3,2-d]pyrimidine (3Bg). Yield 60\%; mp 132-134 ${ }^{\circ} \mathrm{C}$; ${ }^{1} \mathrm{H}$ NMR (DMSO$\left.d_{6}\right) \delta 8.68$ (s, 1H, pyrimidine), $7.92(\mathrm{~d}, 1 \mathrm{H}, J=1.6 \mathrm{~Hz}, \mathrm{H}-3$, pyrazole), $7.82(\mathrm{~d}, 1 \mathrm{H}, J=5.9 \mathrm{~Hz}$, thiophene), $7.59(\mathrm{~d}, 1 \mathrm{H}, J$ $=5.9 \mathrm{~Hz}$, thiophene), $7.44\left(\mathrm{~s}, 1 \mathrm{H}\right.$, phenyl, $\left.\mathrm{H}_{2}{ }^{\prime}\right), 7.42-7.36(\mathrm{~m}$, $3 \mathrm{H}), 6.78$ (d, 1H, $J=1.6 \mathrm{~Hz}, \mathrm{H}-4$, pyrazole); MS (ESI): $(\mathrm{m} / \mathrm{z})$ $312.95\left(\mathrm{M}^{+}\right)$. Anal. Calcd. For $\mathrm{C}_{15} \mathrm{H}_{9} \mathrm{ClN}_{4} \mathrm{~S}$ : C, 57.60; H, 2.90; N, 17.91. Found: C, 57.46; H, 2.80; N, 17.82 .

4-(5-4-Chlorophenyl-1H-pyrazol-1-yl)thieno[3,2- $d]$ pyrimidine (3Bh). Yield 55\%; mp 92-94 ${ }^{\circ} \mathrm{C} ;{ }^{1} \mathrm{H}$ NMR (DMSO$\left.d_{6}\right) \delta 8.66(\mathrm{~s}, 1 \mathrm{H}$, pyrimidine $), 8.06(\mathrm{~d}, 1 \mathrm{H}, J=5.9 \mathrm{~Hz}$, thiophene), 7.90 (d, 1H, $J=1.6 \mathrm{~Hz}, \mathrm{H}-3$, pyrazole), 7.60 (d, $1 \mathrm{H}, J=5.9 \mathrm{~Hz}$, thiophene), $7.33(\mathrm{~d}, 2 \mathrm{H}$, phenyl), $7.21(\mathrm{~d}, 2 \mathrm{H}$, phenyl), 6.50 (d, 1H, $J=1.6 \mathrm{~Hz}, \mathrm{H}-4$, pyrazole); MS (ESI): $(\mathrm{m} / z) 312.98\left(\mathrm{M}^{+}\right)$. Anal. Calcd. For $\mathrm{C}_{15} \mathrm{H}_{9} \mathrm{ClN}_{4} \mathrm{~S}$ : C, 57.60; H, 2.90; N, 17.91. Found: C, 57.76; H, 2.80; N, 17.80 .

4-(5-Phenyl-1 $\boldsymbol{H}$-pyrazol-1-yl)thieno[2,3-d]pyrimidine (4Aa). ${ }^{1} \mathrm{H}$ NMR (DMSO- $d_{6}$ ) $\delta 8.96$ (s, $1 \mathrm{H}$, pyrimidine), 8.60 (d, $1 \mathrm{H}, J=5.9 \mathrm{~Hz}$, thiophene), 7.97-7.94 (m, 3H, phenyl and pyrazole), 7.62 (d, 1H, $J=5.9 \mathrm{~Hz}$, thiophene), 7.50-7.47 (m, $3 \mathrm{H}$, phenyl), 7.10 (d, 1H, $J=2.8 \mathrm{~Hz}, \mathrm{H}-4$, pyrazole); MS (ESI): $(m / z) 278.88\left(\mathrm{M}^{+}\right)$. Anal. Calcd. For $\mathrm{C}_{15} \mathrm{H}_{10} \mathrm{~N}_{4} \mathrm{~S}$ : C, 64.73; H, 3.62; N, 20.13. Found: C, 64.60; H, 3.55; N, 20.03.

4-((Z)-2-((E)-1-(4-Bromophenyl)-3-(2-(thieno[3,2-d]pyramid-in-4-yl)hydrazinyl)allylidene)hydrazinyl)thieno[3,2d]pyramid-ine (5Bf). ${ }^{1} \mathrm{H}$ NMR (DMSO- $d_{6}$ ) $\delta 10.92(\mathrm{~s}, 1 \mathrm{H}$, $\mathrm{NH})$, 8.69-8.67 (m, 2H, pyrimidine), 8.47-8.44 (m, 2H, thiophene), $7.73(\mathrm{~d}, 2 \mathrm{H}$, phenyl), 7.60 (d, 2H, phenyl), 7.50-
7.47 (m, 2H, thiophene), $7.12(\mathrm{~m}, 1 \mathrm{H}), 6.14$ (m, 1H, phenyl); MS (ESI): $(m / z) 524.74\left(\mathrm{M}^{+}\right)$. Anal. Calcd. For $\mathrm{C}_{21} \mathrm{H}_{15} \mathrm{BrN}_{8} \mathrm{~S}_{2}$ : C, 48.19; H, 2.89; N, 21.41. Found: C, 48.03; H, 2.78; N, 21.23.

Acknowledgments. This work was supported by the Korea Research Foundation (project number 2010-0021038).

\section{References}

1. Feldmann, M.; Brennan, F. M.; Maini, R. N. Annu. Rev. Immunol. 1996, 14, 397

2. Jones, S. A.; Scheller, J.; Rose-John, S. J. Clin. Invest. 2011, 121, 3375 .

3. Aaronson, D.; Horvath, C. Science 2002, 296, 1653.

4. Yu, H.; Pardoll, D.; Jove, R. S. Nat. Rev. Cancer 2009, 9, 798.

5. (a) Rose-John, S.; Waetzig, G. H.; Schller, J.; Gratzinger, J.; Seegert, D. Expert Opin. Ther. Targets 2007, 11, 613. (b) Adachi, Y.; Yoshio-Hoshino, N.; Nishimoto, N. Curr. Pharm. Des. 2008, 14, 1217.

6. (a) Hafez, H. N.; El-Gazzara, A. B. A. Bioorg. Med. Chem. Lett. 2008, 18, 5222. (b) Alagarsamy, V.; Meena, S.; Ramseshu, K. V.; Solomon, V. R.; Thirumurugan, K.; Dhanabal, K.; Murugan, M. Eur. J. Med. Chem. 2006, 41, 293.

7. (a) Whang, J.; Song, Y.-H. J. Heterocycl. Chem. 2013, 50, 603. (b) Lee, H. J.; Kim, S. M.; Song, Y.-H. Heterocycl. Commun. 2013, 19, 101. (c) Whang, J.; Song, Y.-H. Heterocycles 2012, 85, 155. (d) Song, Y.-H.; Son, H. Y. J. Heterocycl. Chem. 2011, 48, 597. (e) Song, Y.-H.; Moon, J. Heterocycl. Commun. 2011, 17, 135. (f) Song, Y.-H.; Son, H. Y. J. Heterocycl. Chem. 2010, 47, 1183. (g) Son, H. Y.; Song, Y.-H. Bull. Korean Chem. Soc. 2010, 31, 2242. (h) Song, Y.-H.; Jo, B. S. J. Heterocycl. Chem. 2009, 46, 1132. (i) Jo, B. S.; Son, H. Y.; Song, Y.-H. Heterocycles 2008, 75, 3091.

8. Rho, M. C.; Song, Y.-H.; Lee, S. W.; Park, C. S.; Oh, H. M. Korean Patent, appl. 10-2013-0008307, 2013.

9. Yet, L. In Progress in Heterocyclic Chemistry; Gribble, G. W., Joule, J. A., Eds.; Elsvier: Oxford, 2009; Vol. 20, Chapter 5.4, pp 190-219.

10. Rashad, A. E.; Mahmoud, A. E.; Ali, M. M. Eur. J. Med. Chem. 2011, 46, 1019.

11. Zora, M.; Kivrak, A. J. Org. Chem. 2011, 76, 9379. The recent references on pyrazole synthesis are therein.

12. Xu, Z. L.; Li, H. X.; Ren, Z. G.; Du, W. Y.; Xu, W. C.; Lang, J. P. Tetrahedron 2011, 67, 5282.

13. Lee, S. W.; Yun, B. R.; Kim, M. H.; Park, C. S.; Lee, W. S.; Oh, H. M.; Rho, M. C. Planta Med. 2012, 78, 903.

14. Li, H. C.; Zhang, G. Y. Acta Pharmacol. Sin. 2003, 24, 1131. 